\title{
SWIR-LWIR Photoluminescence from Sb-based Epilayers Grown on GaAs Substrates by using MBE
}

\author{
Laiq Hussain and Håkan PetTersson* \\ Department of Mathematics, Physics and Electrical Engineering, \\ Halmstad University, Halmstad S-30118, Sweden and \\ NanoLund and Solid State Physics, Lund University, Lund S-22100, Sweden \\ Qin Wang, Amir Karim and Jan Anderson \\ Acreo Swedish ICT AB, Kista 16425, Sweden \\ Mehrdad JAFARI \\ Department of Mathematics, Physics and Electrical Engineering, Halmstad University, Halmstad S-30118, Sweden \\ Jindong Song, Won Jun ChOI, Il Ki Han and Ju Young Lim \\ Center for Opto-Electronic Convergence Systems, \\ Korea Institute of Science and Technology, Seoul 02792, Korea
}

(Received 8 March 2018, in final form 19 June 2018)

\begin{abstract}
Utilizing Sb-based bulk epilayers on large-scale low-cost substrates such as GaAs for fabricating infrared (IR) photodetectors is presently attracting significant attention worldwide. For this study, three sample series of $\mathrm{GaAs}_{x} \mathrm{Sb}_{1-x}, \mathrm{In}_{1-x} \mathrm{Ga}_{x} \mathrm{Sb}$, and $\mathrm{InAs}_{x} \mathrm{Sb}_{1-x}$ with different compositions were grown on semi-insulating GaAs substrates by using molecular beam epitaxy (MBE) and appropriate InAs quantum dots (QDs) as a defect-reduction buffer layer. Photoluminescence (PL) signals from these samples were observed over a wide IR wavelength range from $2 \mu \mathrm{m}$ to $12 \mu \mathrm{m}$ in agreement with the expected bandgap, including bowing effects. In particular, interband PL signals from $\mathrm{InAs}_{x} \mathrm{Sb}_{1-x}$ and $\mathrm{In}_{1-x} \mathrm{Ga}_{x} \mathrm{Sb}$ samples even at room temperature show promising potential for IR photodetector applications.
\end{abstract}

PACS numbers: 81.05.Ea

Keywords: SWIR, MWIR, LWIR, Sb-based thin films, IR detector

DOI: $10.3938 /$ jkps.73.1604

\section{INTRODUCTION}

High-temperature operating low-cost infrared (IR) photodetectors sensitive to short-wave infrared (SWIR), mid-wave infrared (MWIR) and long-wave infrared (LWIR) radiation covering the spectral range from $1.4 \mu \mathrm{m}$ to $15 \mu \mathrm{m}$ are very attractive for various imaging and sensing applications [1]. State-of-the-art MWIR and LWIR photodetectors are presently dominated by HgCdTe (MCT) detectors and GaAs/AlGaAs quantumwell infrared photodetectors (QWIPs). While MCT detectors on one hand display an excellent quantum efficiency, they are very expensive and suffer from issues related to inhomogeneous material properties over large detector arrays. QWIPs on the other hand suffer from limited quantum efficiency. Therefore, new IR pho- todetector technologies are being explored worldwide for the next generation of IR imaging/sensing systems with higher operating temperatures and lower cost. For example, type I- and type-II quantum-dot photodetectors (QDIPs) and type-II InAs/GaSb-based strained layer superlattice (T2SL) photodetectors are two such promising candidates [2-4]. However, achieving the desired cost and performance figure of merits for these IR photodetectors is still challenging.

Considerable interest in the development of novel IIIV IR detectors exists due to available straightforward fabrication techniques and relatively low cost substrates. For example, utilizing 4-in and 6-in GaAs substrates with appropriate buffer layers to facilitate growth of various novel quantum structures can promote the proof-ofconcept prototypes demonstrated in research laboratory settings to general semiconductor production.

Ternary III-V semiconductors based on InAs, GaSb,

*E-mail: hakan.pettersson@hh.se 


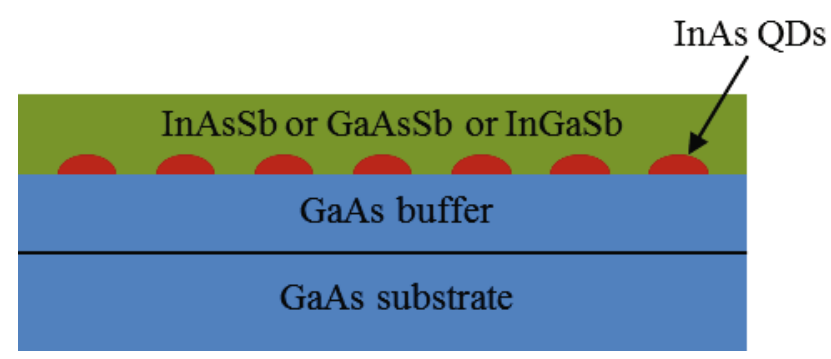

Fig. 1. (Color online) Schematic of the sample structure.

$\mathrm{AlSb}$ and InSb with different compositions can form an approximately lattice-matched set of epilayers covering a wide range of energy gaps. In particular, their extraordinarily large bowing dependence of the bandgap on the composition makes them suitable as detector materials in the MWIR or the LWIR region. The III$\mathrm{V}$ ternary alloy $\mathrm{InAs}_{x} \mathrm{Sb}_{1-x}$ has the lowest energy gap among the III-V semiconductors. For example, the bandgap of $\mathrm{InAs}_{0.35} \mathrm{Sb}_{0.65}$ is around $100 \mathrm{meV}$, corresponding to $12.4 \mu \mathrm{m}$, due to the strong bandgap bowing effect [5]. Further advantages of this material system compared to the MCT system include better stability during growth, accessible low-cost substrates and higher room-temperature electron mobility $[6,7]$.

Although epitaxy of Sb-based materials on GaAs substrates is a promising alternative, the quality of the epilayers must be maintained which is challenging due to the lattice mismatch between the epilayers and the substrates. For instance, the $\operatorname{InAs}_{x} \mathrm{Sb}_{1-x}$ ternary alloys are mismatched to GaAs by between $7.2 \%$ and $14.5 \%$, depending on composition. The substantial compressive strain between the epilayer and the substrate induces various kinds of defects. The problem can be reduced by incorporation of thin strained layers or by the growth of thicker buffer layers with or without graded lattice parameters. Extensive structural, electrical and optical characterizations are needed to study the crystalline quality of the grown epilayers. Such analyses provide valuable feedback for revealing the growth parameters necessary for reliable device processing.

This work includes the growth and the characterization of three sample series of $\mathrm{GaAs}_{x} \mathrm{Sb}_{1-x}, \mathrm{In}_{1-x} \mathrm{Ga}_{x} \mathrm{Sb}$ and $\mathrm{InAs}_{x} \mathrm{Sb}_{1-x}$ with different compositions grown on semi-insulating (SI) GaAs substrates by using MBE. Their structural properties were characterized by using high-resolution X-ray diffraction (HRXRD), scanning electron microscopy (SEM) and transmission electron microscopy (TEM). The optical properties of the samples were investigated using photoluminescence (PL) spectroscopy at different temperatures from $77 \mathrm{~K}$ to $300 \mathrm{~K}$. The PL signal was recorded by using a Bruker V70 Fourier transform infrared (FTIR) spectrometer system equipped with liquid-nitrogen-cooled InSb and MCT detectors. A diode-pumped solid-state (DPSS) Cobolt $532 \mathrm{~nm}$ laser was invoked for optical pumping.

\section{GROWTH OF SB-BASED EPILAYERS ON GAAS BY USING MBE}

The samples investigated in this work, schematically shown in Fig. 1, were all grown on (001) SI-GaAs substrates in a Riber compact 21E solid source MBE system with a rotating sample stage for uniform film growth. The surface oxide was removed from the GaAs substrates by heating the samples to a substrate temperature $T_{s}=620{ }^{\circ} \mathrm{C}$ under the $\mathrm{As}_{2}$ mode. A approximately $\sim 200 \mathrm{~nm}$ thick GaAs buffer layer was consecutively grown at $T_{s}=580{ }^{\circ} \mathrm{C}$. The temperature of the substrates was thereafter lowered to $480{ }^{\circ} \mathrm{C}$ for the growth of InAs QDs with a thickness corresponding to about 3 monolayers by using the Stranski-Krastanov growth mode. After growth of the QD layer, the samples were annealed for $1 \mathrm{~min}$. The InAs QD layer acts as an intermediate layer to reduce defects caused by the substantial lattice mismatch between the GaAs substrates and the Sb-based epilayers [8]. Because InAs is a material with a lattice parameter between that of GaAs and that of the $\mathrm{GaAs}_{x} \mathrm{Sb}_{1-x}, \operatorname{InAs}_{x} \mathrm{Sb}_{1-x}$ or $\mathrm{In}_{1-x} \mathrm{Ga}_{x} \mathrm{Sb}$ epilayer grown in this work, the use of InAs QDs as an intermediate layer is a viable alternative for overcoming latticemismatch challenges between the epilayers and the GaAs substrates. Moreover, QDs can interrupt the dislocations extending from the epilayer/substrate interface. The dislocation lines that have an in-plane component may loop around the QDs and no longer extend the sample surface, or the dislocations may terminate at the surfaces of the dots.

For the final growth of the Sb-epilayers, the substrate temperature was carefully optimized. For example, $400{ }^{\circ} \mathrm{C}, 480{ }^{\circ} \mathrm{C}$, and $470{ }^{\circ} \mathrm{C}$ were selected for $\mathrm{InSb}$, InAs, GaSb, respectively. For intermediate $\mathrm{Sb}$ compositions, linearly interpolated temperatures were chosen. The samples exhibited streaky reflection highenergy electron diffraction (RHEED) patterns during the latter part of the growth indicating laminar growth. Besause the thickness of the Sb-based epilayers $(\sim 1 \mu \mathrm{m})$ was always larger than the critical thickness on GaAs, the epilayers were fully relaxed thus all collected optical data were unaffected by the strain.

The proper formation of InAs QDs on the GaAs buffer layer was confirmed by using atomic force microscopy $(\mathrm{AFM})$ on a reference sample. The results show that the density of QDs was about $6.0 \times 10^{10} / \mathrm{cm}^{2}$, with the average height and width being $4.9 \pm 1.5 \mathrm{~nm}$ and $37.9 \pm$ $6.4 \mathrm{~nm}$, respectively.

An interesting comparison can be made between the $\mathrm{InSb}$ layers grown on $\mathrm{GaAs}$ and the samples with $\mathrm{InSb}$ layers grown on InSb substrates. In Ref. [8], the authors showed that $2.6 \mu \mathrm{m}$ thick InSb layers grown on GaAs by using an InAs QD defect reduction layer exhibit an electron mobility of $67,890 \mathrm{~cm}^{2} / \mathrm{Vs}$ at $300 \mathrm{~K}$. In another report, the mobility of $\sim 6 \mu \mathrm{m}$ thick InSb layers grown on GaAs by using a similar QD defect reduction 


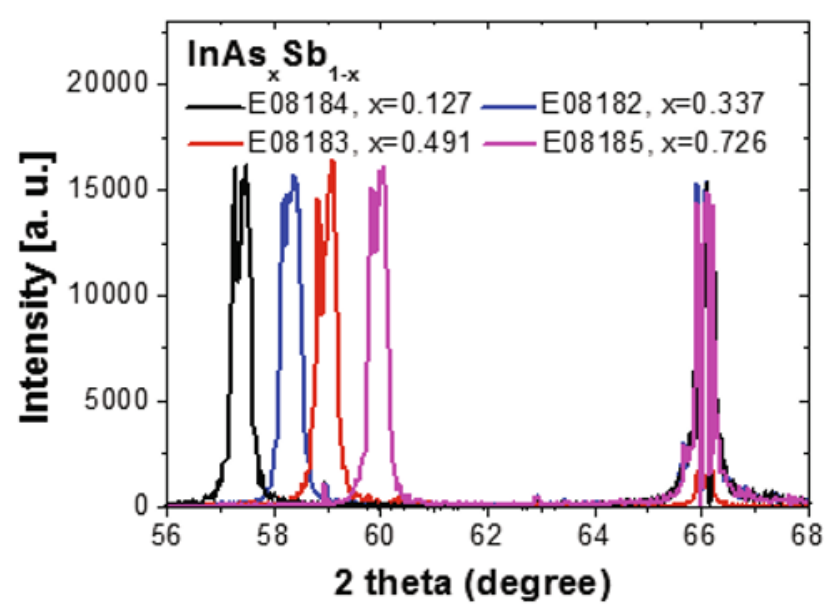

Fig. 2. (Color online) HRXRD spectra of $\operatorname{InAs}_{x} \mathrm{Sb}_{1-x}$ samples grown on GaAs substrates by using MBE. The two observed peaks for each ternary alloy are caused by the two present wavelengths $\mathrm{K}_{\alpha 1}$ and $\mathrm{K}_{\alpha 2}$ from the $\mathrm{Cu}$-based XRD source. The authors considered only $\mathrm{K}_{\alpha 1}$ in the calculations.

layer were $\sim 78,000 \mathrm{~cm}^{2} / \mathrm{Vs}$ at $300 \mathrm{~K}[9]$, which is comparable to that of InSb layers grown on InSb substrates $\left(\sim 80,000 \mathrm{~cm}^{2} / \mathrm{Vs}\right.$ at $\left.300 \mathrm{~K}\right)$. These results indicate that high quality InSb layers were grown on GaAs by using the proposed defect reduction technique.

A 2 to $3 \mu \mathrm{m}$ thick buffer layer is needed to grow highquality InSb epilayers on GaAs. This requires at least 2 - 3 hours of additional growth time and correspondingly higher running costs compared to the growth of InSb layers on InSb or GaSb substrates. The cost of 2 -inch GaAs substrates is typically less than 100 USD /EA. This price is $\sim 1 / 10$ of corresponding price for an $\mathrm{InSb}$ or GaSb substrates. In summary, the expected cost and the performance figure of merit amounts to at least $4 \sim 5$ in favor of our proposed growth method. Moreover, while 6-inch GaAs substrates are available on the market, 3 -inches is the maximum size of GaSb wafers. Furthermore, in contrast to the case of GaAs, semi-insulating $\mathrm{InSb}$ and $\mathrm{GaSb}$ substrates are not available. This is a critical issue for electrical device applications. The authors are presently working on developing the technology to grow Sb-based materials on large-area Si substrates in line with the "More than Moore" concept [10].

1. Structural properties of $\operatorname{InAs}_{x} \mathrm{Sb}_{1-x}$ epilayers

InAs $\mathrm{Sb}_{1-x}$ ternary alloys have received increasing attention due to important applications, such as $\mathrm{InAs}_{x} \mathrm{Sb}_{1-x}$ infrared photodetectors operating at room temperature $[11,12]$. These alloys typically also exhibit small compositional variations and thus small bandgap changes over large device areas. A series of $\operatorname{InAs}_{x} \mathrm{Sb}_{1-x}$ ternary alloy samples with $x=0.000,0.127,0.337,0.491$,

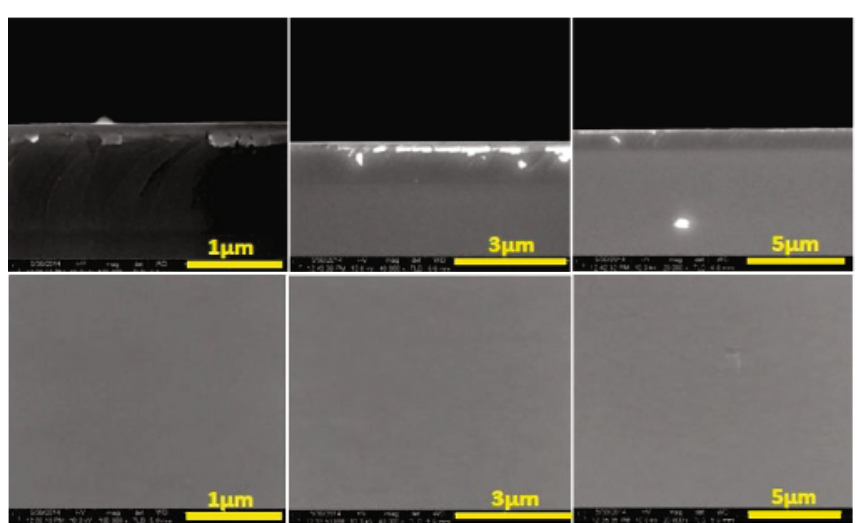

Fig. 3. (Color online) Cross-sectional (upper panel) and top-view (lower panel) SEM images of an $\mathrm{InAs}_{0.726} \mathrm{Sb}_{0.274}$ sample.

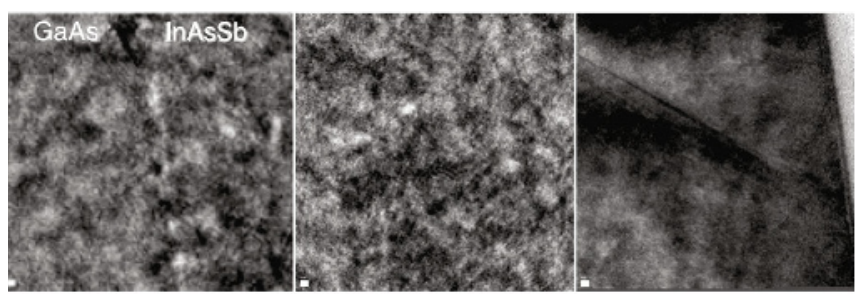

Fig. 4. Cross-sectional TEM results recorded at (a) the interface between the GaAs buffer layer and the InAs $\mathrm{A}_{0.726} \mathrm{Sb}_{0.274}$ epilayer and at the (b) middle and the (c) top of the epilayer. The scale bar (left bottom) is $10 \mathrm{~nm}$.

0.726 and 1.000 were grown on (001) GaAs substrates by using the growth process described above. The composition was determined by using HRXRD as shown in Fig. 2. As well known, the (400) rocking curve can be used to evaluate the film quality. The peaks at diffraction angles between $56^{\circ}$ and $61^{\circ}$, shown in Fig. 2, are the (400) diffraction peaks of the $\operatorname{InAs}_{x} \mathrm{Sb}_{1-x}$ epilayers with different compositions. Evidently, no other crystal structures are resolved. The full width at half maximum (FWHM) of the (400) peaks is similar to that of $\operatorname{InAs}_{0.23} \mathrm{Sb}_{0.77}$ grown on a GaSb, InAs or InSb substrate [11-13]. The peak at a diffraction angle of about $66^{\circ}$ is due to the single-crystal GaAs substrate.

The thickness of the epilayers is about $1 \mu \mathrm{m}$, which was controlled by using the growth rate and growth time and was characterized by using cross-sectional SEM. Figure 3 shows a series of SEM images of an $\operatorname{InAs}_{x} \mathrm{Sb}_{1-x}$ sample with $x=0.726$ (E08185). Evidently, the boundary between the epilayer and the GaAs substrate is flat and straight, and the top view images indicate a smooth surface.

The cross-sectional structures and morphologies of the samples were also analyzed by using TEM and highresolution scanning TEM (HR-STEM) with an FEI Titan 80 - 300 unit (with monochromatic and Cs-corrected probe) operating at $300 \mathrm{keV}$. Figures 4 shows the crosssectional TEM results from which the defect density in 

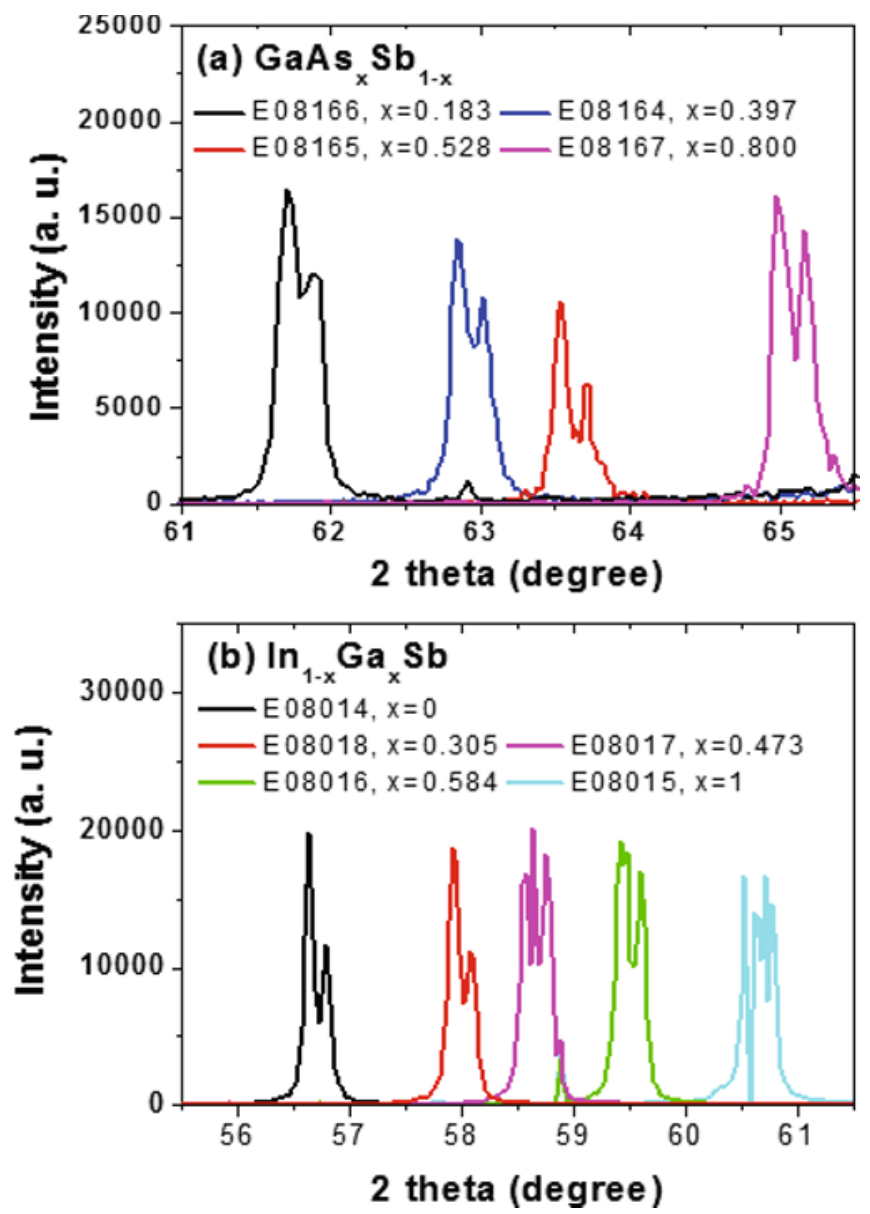

Fig. 5. (Color online) HRXRD spectra of (a) $\mathrm{GaAs}_{x} \mathrm{Sb}_{1-x}$ and (b) $\operatorname{In}_{1-x} \mathrm{Ga}_{x}$ Sb epilayers.

the epilayer is clearly reduced with increasing distance from the interface with the GaAs buffer layer. The remaining line density of threading dislocations and twins at the surface is merely $\sim 4$ defects $/ \mu \mathrm{m}$.

\section{2. $\mathrm{GaAs}_{x} \mathrm{Sb}_{1-x}$ and $\operatorname{In}_{1-x} \mathrm{Ga}_{x} \mathrm{Sb}$ epilayers}

$\mathrm{GaAs}_{x} \mathrm{Sb}_{1-x}$ and $\mathrm{In}_{1-x} \mathrm{Ga}_{x} \mathrm{Sb}$ ternary alloys show promising performance for SWIR and MWIR detectors. By varying their composition, the detection peak wavelength can be tuned from $0.85 \mu \mathrm{m}$ (GaAs) to about $5.5 \mu \mathrm{m}$ (InSb). The present Sb-based epilayers were again grown on (001) GaAs substrates by using MBE with the growth method described above. The layers are about $0.7 \mu \mathrm{m}$ thick, which is well beyond their critical thicknesses, so the data presented below are characteristics of strain-relaxed materials. The compositions were revealed using HRXRD and the results are shown in Fig. 5. The peaks in Fig. 5(a) at diffraction angles between $61^{\circ}$ and $65.5^{\circ}$ are the (400) diffraction peaks of $\mathrm{GaAs}_{x} \mathrm{Sb}_{1-x}$ epilayers with the different compositions $x=0.183,0.397,0.528$ and 0.800 , respectively. The peaks in Fig. 5 (b) at diffraction angles from $55.5^{\circ}$ to $61.5^{\circ}$ are similarly the (400) diffraction peaks of $\mathrm{In}_{1-x} \mathrm{Ga}_{x} \mathrm{Sb}$ epilayers with compositions $x=1,0.584$, $0.473,0.305$ and 0 , respectively.

Evidently, the sharpness and the FWHM of the diffraction peaks of the samples vary, which indicates differences in the crystal quality of the epilayers. Here, we should mention that the growth parameters were not fully optimized for every individual sample. The diffraction peaks from the GaAs substrate were also observed for these samples, but they are not shown in the plots.

\section{RESULTS AND DISCUSSION}

\section{PL measurements}

Photoluminescence spectra of the samples were obtained using an FTIR spectrometer equipped with an InSb or MCT detector and step-scan functionality with lock-in detection. A solid-state laser emitting at $532 \mathrm{~nm}$ was used for optical excitation. The temperature and the laser power dependences in the range from $70 \mathrm{~mW}$ to $400 \mathrm{~mW}$ were investigated in detail.

\section{PL in the LWIR regime from $\operatorname{InAs} s_{x} \mathrm{Sb}_{1-x}$} epilayers

Figure 6(a) shows PL spectra of the $\mathrm{InAs}_{x} \mathrm{Sb}_{1-x}$ samples, recorded by using an MCT detector under a $365 \mathrm{~mW}$ excitation power at $77 \mathrm{~K}$. For the $\mathrm{InAs}_{x} \mathrm{Sb}_{1-x}$ layers with $\mathrm{Sb}$ compositions of $27 \%$ (E08185), $51 \%$ (E08183) and $66 \%$ (E08182), PL peaks were found at wavelengths of $6.5 \mu \mathrm{m}, 8.9 \mu \mathrm{m}$ and $10.5 \mu \mathrm{m}$, respectively. The results, thus, reveal a clear trend of a redshift in peak wavelength with increasing Sb composition. The FWHM of the PL peak from sample E08183 with $51 \% \mathrm{Sb}$ and sample E08182 with $66 \% \mathrm{Sb}$ is clearly much broader than that of sample E08185 with $27 \% \mathrm{Sb}$, indicating larger compositional variations in samples with higher Sb contents. A change trend is observed for sample E08184 with $87 \%$ Sb, which exhibits a PL peak wavelength shifting towards shorter wavelengths. The extracted PL peak position at $T=77 \mathrm{~K}$ as a function of the composition are plotted as red dots in Fig. 6(b). These results can be compared to the expected bandgap $\left(E_{\mathrm{g}}\right)$ of corresponding $\mathrm{InAs}_{x} \mathrm{Sb}_{1-x}$ ternary alloys. The bandgap of $\mathrm{A}_{x} \mathrm{~B}_{1-x} \mathrm{C}$ compounds, taking into account the bowing effect, is generally given

$E_{\mathrm{g}}\left(\mathrm{A}_{x} \mathrm{~B}_{1-x} \mathrm{C}\right)=E_{\mathrm{g}}(\mathrm{BC})+x\left[E_{\mathrm{g}}(\mathrm{AC})-E_{\mathrm{g}}(\mathrm{BC})\right]-b x(1-x)$,

where $b$ is the bowing parameter [5]. The dependence of the bandgap on the Sb-based ternary composition 

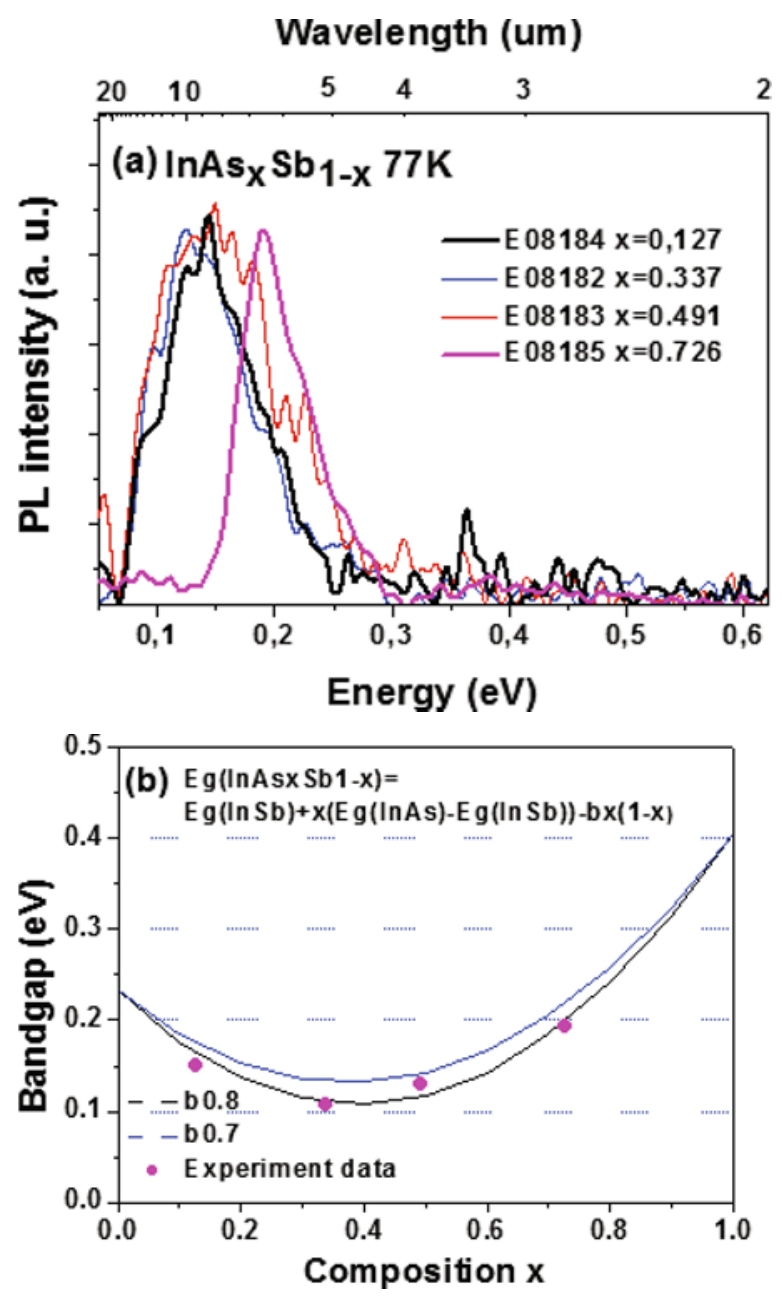

Fig. 6. (Color online) (a) Normalized PL spectra of $\mathrm{InAs}_{x} \mathrm{Sb}_{1-x}$ samples at $77 \mathrm{~K}$. (b) Dependence of the expected bandgap and thus the PL peak energy for two different bowing parameters on the composition $x$ in the $\operatorname{InAs}_{x} \mathrm{Sb}_{1-x}$ epilayers. Red dots are experimental PL data from (a).

is, thus, expected to be non-linear, as is also shown in Fig. 6(b). The bandgaps of InAs and InSb at $T=77 \mathrm{~K}$ are 0.404 and $0.232 \mathrm{eV}$, respectively. The expected bandgap of $\operatorname{InAs}_{x} \mathrm{Sb}_{1-x}$ versus composition is calculated using Eq. (1), and the result is shown for two bowing parameters $b=0.7 \mathrm{eV}$ and $b=0.8 \mathrm{eV}$ [5] in Fig. 6(b). Evidently, our experiential data fit best with a bowing parameter $\mathrm{b}=0.8 \mathrm{eV}$.

As shown in Fig. 7(a), clear PL spectra were observed are a broad temperature range from sample InAs ${ }_{x} \mathrm{Sb}_{1-x}$ $(x=0.726)$. Interestingly, the PL intensity at $300 \mathrm{~K}$ was only decreased by a factor of two as compared to 100 K. No clear red shift in the PL peak position was observed as the temperature is increased. Figures 7(b) and (c) show PL spectra versus optical pumping power at $77 \mathrm{~K}$ and $300 \mathrm{~K}$, respectively. With increasing excitation power, the $\mathrm{PL}$ peak redshifts at $77 \mathrm{~K}$, which possibly reflects a thermal heating of the samples. In
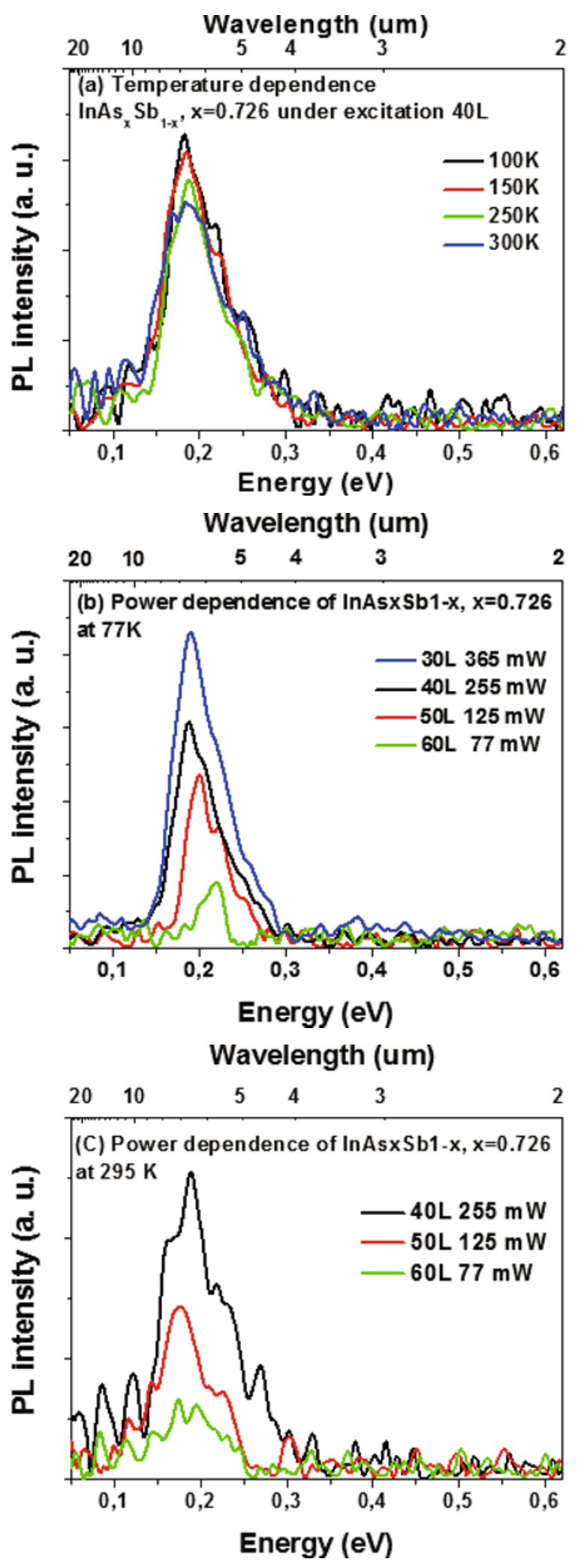

Fig. 7. (Color online) PL spectra of an $\operatorname{InAs}_{x} \mathrm{Sb}_{1-x}(x=$ 0.726) sample. (a) Temperature dependence of the PL signal. (b) and (c) show the PL dependence on laser power at $77 \mathrm{~K}$ and $295 \mathrm{~K}$, respectively. $40 \mathrm{~L}$ and $50 \mathrm{~L}$ and $60 \mathrm{~L}$ represent different apertures that control the excitation power. 

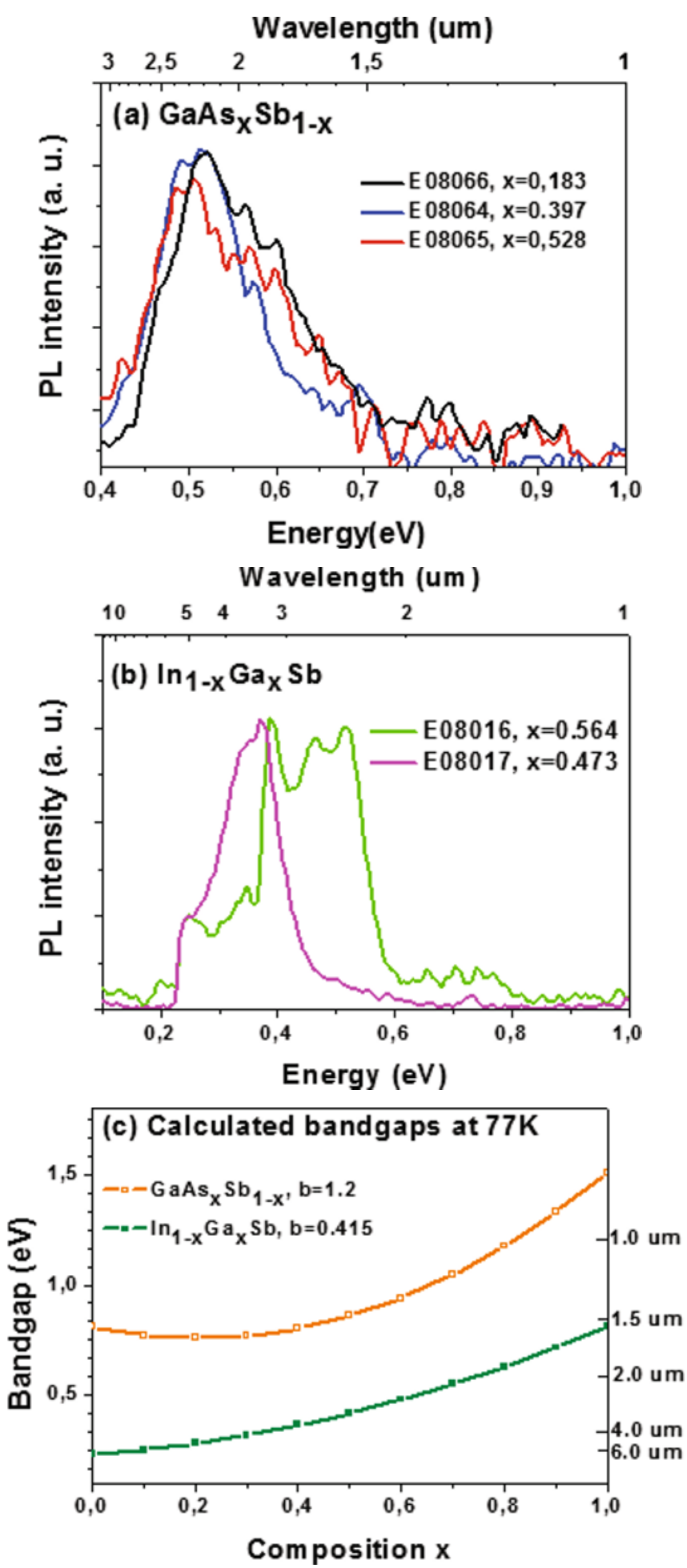

Fig. 8. (Color online) PL spectra of (a) $\mathrm{GaAs}_{x} \mathrm{Sb}_{1-x}$ and (b) $\operatorname{In}_{1-x} \mathrm{Ga}_{x} \mathrm{Sb}$ epilayers measured at $77 \mathrm{~K}$. The color codes are arranged in agreement with those in Figs. 5(a) and (b). (c) Bandgap of the corresponding ternary Sb-based compounds calculated using Eq. (1).

contrast, no clear power dependence of the PL peak position is observed at room temperature. This can be ex- plained by the low signal-to-noise ratio of the PL in this case, which smears out the redshift. The PL intensity clearly increases with increasing optical pumping power at both $77 \mathrm{~K}$ and $295 \mathrm{~K}$. The fact that PL signals can be observed at room temperature indicates a good crystal quality of the InAs $\mathrm{Sb}_{1-x}$ epilayers, which confirms the structural characterization results shown in Figs. 2, 3 and 4 in Sec. II of this paper.

3. PL in SWIR and MWIR regimes from $\mathrm{GaAs}_{x} \mathrm{Sb}_{1-x}$ and $\mathrm{In}_{1-x} \mathrm{Ga}_{x} \mathrm{Sb}$ epilayers

PL spectra of $\mathrm{GaAs}_{x} \mathrm{Sb}_{1-x}$ and $\mathrm{In}_{1-x} \mathrm{Ga}_{x} \mathrm{Sb}$ epilayers were also obtained as shown in Figs. 8(a) and (b), respectively, by using a measurement set-up with an InSb detector as described above. Only some of the samples showed PL signals at $77 \mathrm{~K}$, which could be attributed to non-radiative recombination caused by defects in the epilayers. Interestingly, a PL signal from one $\mathrm{In}_{1-x} \mathrm{Ga}_{x} \mathrm{Sb}$ sample (E08017 with $x=0.473$ ) was observed even at room temperature, which is evidence of the good crystal quality of this sample. The FWHM is large for all these samples, indicating compositional variations among the samples.

The expected bandgaps (and thus PL peak positions) of the $\mathrm{GaAs}_{x} \mathrm{Sb}_{1-x}$ and $\mathrm{In}_{1-x} \mathrm{Ga}_{x} \mathrm{Sb}$ epilayers have been analysed using Eq. (1), and the results are plotted in Fig. 8(c). The corresponding bowing parameters were again taken from Ref. 5 . In comparison to experimental results, the PL peak position of the $\mathrm{In}_{1-x} \mathrm{Ga}_{x} \mathrm{Sb}$ sample E08017 with $x=0.473$ is in good agreement with the corresponding plot in Fig. 8(c). The other $\mathrm{In}_{1-x} \mathrm{Ga}_{x} \mathrm{Sb}$ sample with composition $x=0.564$ displays a blue-shifted $\mathrm{PL}$, in qualitative agreement with Fig. 8(c), although with a significant spectral broadening. However, all the $\mathrm{PL}$ peak wavelengths observed for the $\mathrm{GaAs}_{x} \mathrm{Sb}_{1-x}$ samples were significantly longer than those expected from Fig. 8(c). The origin of this observation is not clear. The reported bandgap bowing parameters of $\mathrm{GaAs}_{x} \mathrm{Sb}_{1-x}$ are quite scattered and, in fact, depend on the composition. Another source of uncertainty has to do with possible immiscibility, e.g., formation of GaAs and GaSb clusters in the epilayer. If the present $\mathrm{GaAs}_{x} \mathrm{Sb}_{1-x}$ epilayers are, in fact, clustered, a modified band structure and band alignment between the GaAs and the GaSb clusters induced by the strain can form type-I heterostructures (Sb content $<0.42$ ) or type-II heterostructures (Sb content $>0.42$ ) [14]. The calculated band gap bowing is 1 $1.2 \mathrm{eV}$ and is associated with the lowest direct band gap $[5,15,16]$. Type-II strain-free GaSb QDs and quantum rings in a GaAs matrix have been to emit PL at $\sim 1.1 \mathrm{eV}$ and $\sim 0.95 \mathrm{eV}$, respectively, at low temperatures [17,18], while a PL peak position of less than $\sim 0.7 \mathrm{eV}$ was expected from calculations [19]. The observed PL peak energy of $\sim 0.5 \mathrm{eV}$ in our case is, thus, far smaller than that expected from any confined $\mathrm{GaAs}_{x} \mathrm{Sb}_{1-x}$ nanostruc- 
tures. The $\sim 0.5 \mathrm{eV}$ PL observed in our case could possibly stem from indirect transitions in such type-II clusters. Similar low-energy PL peaks were also reported in Ref. 20, and were attributed to a corrugation of the GaSb layer. In type-II GaSb/GaAs quantum wells, the $\mathrm{PL}$ peak is strongly shifted to lower energies with respect to the GaSb band gap. If the thickness of the GaSb layer increases, this morphological instability is used finally to leads to the formation of three-dimensional islands (QDs) that can accommodate the strain and, hence, can considerably modify the band offset of type-II GaSb/GaAs heterojunctions [21]. However, in-depth TEM studies are necessary to support this conjecture. The results reveal that further optimization is needed to improve the crystal quality of the $\mathrm{GaAs}_{x} \mathrm{Sb}_{1-x}$ epilayers. In $\mathrm{InAs}_{x} \mathrm{Sb}_{1-x}$, the bandgap energy decreases with increasing Sb composition. For $x=0.80$, the bowing parameter is reported to be about $0.67 \mathrm{eV}$, which is higher than the room temperature value of $0.59 \mathrm{eV}$. The calculated value is $0.70 \mathrm{eV}$ in Ref. 22. The bowing parameter is in the range of 0.67 - $0.69 \mathrm{eV}$ for a very low composition of $\mathrm{Sb}(x=0.91)$ [5]. In Refs. 22 and 23, between the temperatures $77 \mathrm{~K}$ and $300 \mathrm{~K}$, for $\operatorname{InAs}_{x} \mathrm{Sb}_{1-x}$ with $\mathrm{Sb}$ composition close to the one, which we have used, the red-shift is in the range of around $20 \mathrm{meV}$. For the PL measurements, we have used the step-scan mode of FTIR, which clearly provides quite noisy signals, which could be the reason we don't see any considerable red shift in this range.

\section{SUMMARY AND CONCLUSIONS}

We have demonstrated clear PL signals in the SWIR to LWIR wavelength regions from the investigated $\mathrm{In}_{1-x} \mathrm{Ga}_{x} \mathrm{Sb}, \mathrm{GaAs}_{x} \mathrm{Sb}_{1-x}$, and $\mathrm{InAs}_{x} \mathrm{Sb}_{1-x}$ epilayers grown on GaAs by using MBE. The observed PL signals and corresponding bandgap of the epilayers were analysed taking into account the bowing effect. The main research highlight is that the interband PL signals from the $\mathrm{InAs}_{x} \mathrm{Sb}_{1-x}$ and the $\mathrm{In}_{1-x} \mathrm{Ga}_{x} \mathrm{Sb}$ epilayers grown on GaAs substrates were observed even at room temperature, which shows promising potential for high operation temperature IR detector applications.

\section{ACKNOWLEDGMENTS}

This work is partially financed by the Swedish Foundation for International Cooperation in Research and Higher Education (STINT) and by the National Research Foundation of Korea (NRF) through the KoreaSweden Research Cooperation Programme. Also, it has been financed by the Institute Excellence Center IMAGIC, which is led by Acreo Swedish ICT AB. The authors from Korea Institute of Science and Technology (KIST) acknowledge the support from KIST institutional programs, including a flagship program.
Open Access: This article is licensed under a Creative Commons Attribution 4.0 International License, which permits use, sharing, adaptation, distribution and reproduction in any medium or format, as long as you give appropriate credit to the original author(s) and the source, provide a link to the Creative Commons licence, and indicate if changes were made.

The images or other third party material in this article are included in the articles Creative Commons licence, unless indicated otherwise in a credit line to the material. If material is not included in the articles Creative Commons licence and your intended use is not permitted by statutory regulation or exceeds the permitted use, you will need to obtain permission directly from the copyright holder. To view a copy of this licence, visit http://creativecommons.org/licenses/by/4.0/.

\section{REFERENCES}

[1] A. Rogalski, Infrared Physics and Technology. 54, 136 (2011), doi:10.1016/j.infrared.2010.12.003.

[2] A. V. Barve, T. Rotter, Y. Sharma, S. J. Lee, S. K. Noh and S. Krishna, Appl. Phys. Lett. 97, 2008 (2010). doi:10.1063/1.3475022.

[3] O. Gustafsson, A. Karim, C. Asplund, Q. Wang, T. Zabel, S. Almqvist, S. Savage, J. Y. Andersson and M. Hammar, Infrared Physics and Technology 61, 319 (2013), doi:10.1016/j.infrared.2013.09.009.

[4] H. Martijn, C. Asplund, R. M. von Würtemberg and H. Malm, in Proc. SPIE 8704, Infrared Technology and Applications XXXIX. 8704 87040Z-87040Z-9 (2013), doi: $10.1117 / 12.2016602$.

[5] I. Vurgaftman, J. R. Meyer and L. R. Ram-Mohan, J. Appl. Phys. 89, 5815 (2001), doi:10.1063/1.1368156.

[6] T. J. Kim, J. J. Yoon, S. Y. Hwang, D. E. Aspnes, Y. D. Kim, H. J. Kim, Y. C. Chang and J. D. Song, Appl. Phys. Lett. 95 (2009), doi:11190210.1063/1.3216056.

[7] T. J. Kim, J. J. Yoon, J. S. Byun, S. Y. Hwang, D. E. Aspnes, S. H. Shin, J. D. Song, C. T. Liang, Y. C. Chang, N. S. Barange, J. Y. Kim and Y. D. Kim, Appl. Phys. Lett. 102, 3 (2013), doi:10.1063/1.4795622.

[8] J. Y. Lim, J. D. Song and H. S. Yang, Thin Solid Films 520, 6589 (2012), doi:10.1016/j.tsf.2012.06.077.

[9] S. Joo, T. Kim, S. H. Shin, J. Y. Lim, J. Hong, J. D. Song, J. Chang, H-W. Lee, K. Rhie, S. H. Han, K-H. Shin and M. Johnson, Nature 494, 72 (2013), doi:10.1038/nature11817.

[10] K. H. Yoen, J. D. Song, E. H. Lee, H. J. Jang, M. H. Bae, J. Y. Kim, I. K. Han and W. J. Choi, Materials Research Bulletin 57, 152 (2014), doi:10.1016/j.materresbull.2014.05.040.

[11] Y. Z. Gao, X. Y. Gong, W. Z. Fang, G. H. Wu and Y. B. Feng, 48, 0802021 (2009), doi:10.1143/JJAP.48.080202.

[12] I. Shafir, M. Katz, A. Sher, A. Raizman, A. Zussman and M. Nathan, Semiconductor Science and Technology 25, 45 (2010).

[13] C. H. Sun, Q. W. Wang, F. Qiu, Y. F. Lv, H. Y. Deng, S. H. Hu and N. Dai, Journal of Alloys and Compounds 535, 39 (2012). 
[14] J. B. Wang, S. R. Johnson, S. A. Chaparro, D. Ding, Y. Cao, Y. G. Sadofyev, Y. H. Zhang, J. A. Gupta and C. Z. Guo, Phys. Rev. B - Condensed Matter and Materials Physics 70, 1 (2004).

[15] G. Liu, S. Chuang, S. Park and S. Park, J. Appl. Phys. 88, 5554 (2009).

[16] M. Ferhat, Physica Status Solidi 241, 38 (2004).

[17] J. Tatebayashi, B. Liang, D. A. Bussian, H. Htoon, S. Huang, G. Balakrishnan, V. Klimov, L. R. Dawson and D. L. Huffaker, IEEE Transactions on Nanotechnology 8, 269 (2009).

[18] P. J. Carrington, A. S. Mahajumi, M. C. Wagener, J. R. Botha, Q. Zhuang and A. Krier, Physica B 407, 1493 (2012).

[19] K. Gradkowski, T. J. Ochalski, D. P. Williams, J. Tatebayashi, A. Khoshakhlagh, G. Balakrishnan, E. P. O'Reilly, G. Huyet, L. R. Dawson and D. L. Huffaker,
Journal of Luminescence 129, 456 (2009).

[20] F. Hatami, N. N. Ledentsov, M. Grundmann, J. Böhrer, F. Heinrichsdorff, M. Beer, D. Bimberg, S. S. Ruvimov, P. Werner, U. Gösele, J. Heydenreich, U. Richter, S. V. Ivanov, B. Y. Meltser, P. S. Kop'Ev and Z. I. Alferov, Applied Physics Letters, 67 656-658 (1995).

[21] N. N. Ledentsov, J. Böhrer, M. Beer, F. Heinrichsdorff, M. Grundmann, D. Bimberg, S. V. Ivanov, B. Ya. Meltser, S. V. Shaposhnikov, I. N. Yassievich, N. N. Faleev, P. S. KopEv and Z. I. Alferov, Phys. Rev. B 52, 14058 (1995).

[22] Z. M. Fang, K. Y. Ma, D. H. Jaw, R. M. Cohen and G. B. Stringfellow, J. Appl. Phys. 67, 7034 (1990).

[23] M. Y. Yen, R. People and K. W. Wecht, J. Appl. Phys. 64, 952 (1988). 